\title{
Misalignment Angle Calculation Accuracy Analysis of Three-Axis Stabilized Geostationary Satellite
}

\author{
Jian Shang, Chengbao Liu, Lei Yang*, Zhiqing Zhang*, Jing Wang \\ National Satellite Meteorological Center, China Meteorological Administration, Beijing, China \\ Email: shangjian@cma.gov.cn, *yangl@cma.gov.cn, *zqzhang@cma.gov.cn
}

How to cite this paper: Shang, J., Liu, C.B., Yang, L., Zhang, Z.Q. and Wang, J. (2017) Misalignment Angle Calculation Accuracy Analysis of Three-Axis Stabilized Geostationary Satellite. Journal of Geoscience and Environment Protection, 5, 153-165. https://doi.org/10.4236/gep.2017.512011

Received: November 2, 2017

Accepted: December 23, 2017

Published: December 26, 2017

Copyright $\odot 2017$ by authors and Scientific Research Publishing Inc. This work is licensed under the Creative Commons Attribution International License (CC BY 4.0).

http://creativecommons.org/licenses/by/4.0/

\section{c) (i) Open Access}

\begin{abstract}
The most challenging problem of navigation in three-axis stabilized geostationary satellite is accurate calculation of misalignment angles, deduced by orbit measurement error, attitude measurement error, thermal elastic deformation, time synchronization error, and so on. Before the satellite is launched, the misalignment model must be established and validated. But there were no observation data, which is a non-negligible risk of yielding the greatest returns on investment. On the basis of misalignment modeling using landmarks and stars, which is not available between different organizations and is developed by ourselves, experimental data are constructed to validate the navigation processing flow as well as misalignment calculation accuracy. In the condition of using landmarks, the maximum misalignment calculation errors of roll, pitch, and yaw axis are 2, 2, and 104 micro radians, respectively, without considering the accuracy of image edge detection. While in the condition of using stars, the maximum errors of roll, pitch, and yaw axis are 1, 1, and 3 micro radians, respectively, without considering the accuracy of star center extraction. Results are rather encouraging, which pave the way for high-accuracy image navigation of three-axis stabilized geostationary satellite. The misalignment modeling as well as calculation method has been used in the new generation of geostationary meteorological satellite in China, FY-4 series, the first satellite of which was launched at the end of 2016.
\end{abstract}

\section{Keywords}

Misalignment Angle, Accuracy Analysis, Landmark Navigation, Star Navigation, Three-Axis Stabilization Satellite

\section{Introduction}

The development of three-axis stabilized geostationary satellites is the trend of 
geostationary satellites, which has tremendous advantages over spin-stabilized satellites in observation efficiency and operation flexibility [1]. However, three-axis stabilized attitude control mode brings great challenge to image navigation and registration (INR) compared with spin-stabilized satellite, which can satisfy the navigation requirement just by using edge detection of the earth's disk [2]. That's because a spin stabilized satellite tends to equalize the thermal variation seen by the instrument over the day, whereas the thermal gradients across the three-axis stabilized platform are more extreme [3]. This can introduce thermal distortions in the platform structure causing changes in the instrument to platform alignment, which will result in navigation error. And this makes the navigation of three-axis stabilized satellite more complicated, and more precise navigation method shall be put into use.

INR is the engineering discipline that deals with the problem of calibrating and stabilizing image geometry [4]. Without accurate navigation, the satellite products could not be retrieved accurately. For three-axis stabilized geostationary satellites, the misalignment caused by thermal elastic deformation, which can't be measured directly, is the most difficult element to model and the greatest challenge of INR. The misalignment must be calculated and forecasted accurately in the ground system. With uploaded coefficients, compensation could be accomplished by the on-board system. Navigation is fulfilled by complicated satellite-earth operation [5]. Studies about navigation process have been carried out for American GOES satellites, European MTG satellites, and so on [3] [6]. But the misalignment modeling and calculating technique is so critical that there are seldom fully open reports and articles describing the methods in detail.

On account of the great image navigation risk, experimental data must be simulated to support analysis before the satellite's launch. In this paper, effective experimental data are constructed to validate the navigation processing flow as well as misalignment calculation accuracy, based on misalignment modeling. Landmark and star navigations are both realized, which are indispensable to high-accuracy navigation for three-axis stabilized geostationary satellites, including meteorological satellites, earth resource satellites, military satellites, and so on.

\section{Misalignment Angle Calculation and Experimental Data Construction}

\subsection{Misalignment Modeling}

Two navigation methods are considered for new generation three-axis stabilized geostationary satellite, one uses landmarks and the other uses stars. The former takes the errors of the whole satellite-to-ground link into consideration, while the latter can achieve higher accuracy. This technique is so critical in geolocation of three-axis stabilized geostationary satellite that there are not any reports and articles describing the methods in detail.

1) Landmark navigation 
The errors of whole satellite-to-ground link in geostationary satellite system, including time synchronization error, orbit determination and prediction error, attitude measurement error, calculation error of star navigation, and so on, impact navigation accuracy. Landmark navigation can correct navigation error systematically, which makes it indispensible in image navigation. The primary steps of landmark navigation are proposed as follows, which is shown in Figure 1.

a) Select proper landmarks, according to the distribution, cloud cover, observation time, etc.

b) Generate observation instructions corresponding to each selected landmark, and upload to the satellite.

c) The spaceborne instruments carry out observations of the selected landmarks, and observation data are transmitted to the ground.

d) Implement automated landmark matching and obtain the deviated position of each effective landmark [7] [8].

e) Calculate misalignment angles using landmarks' deviations and ideal positions.

f) Finish accurate navigation calculation using misalignment angles, orbit data, attitude data, etc.

According to observation geometry of navigation equation, the actual view vector $\boldsymbol{d}$ and the idea view vector $\hat{\boldsymbol{d}}$ of a landmark are both described in earth centered rotating coordinate system (ECR). The view vectors need to be rotated to orbital coordinate system (ORB) [9].

$$
\boldsymbol{d}_{\text {orb }}=T_{\mathrm{ECI2ORB}} T_{\mathrm{ECR} 2 \mathrm{ECI}} \hat{\boldsymbol{d}}
$$

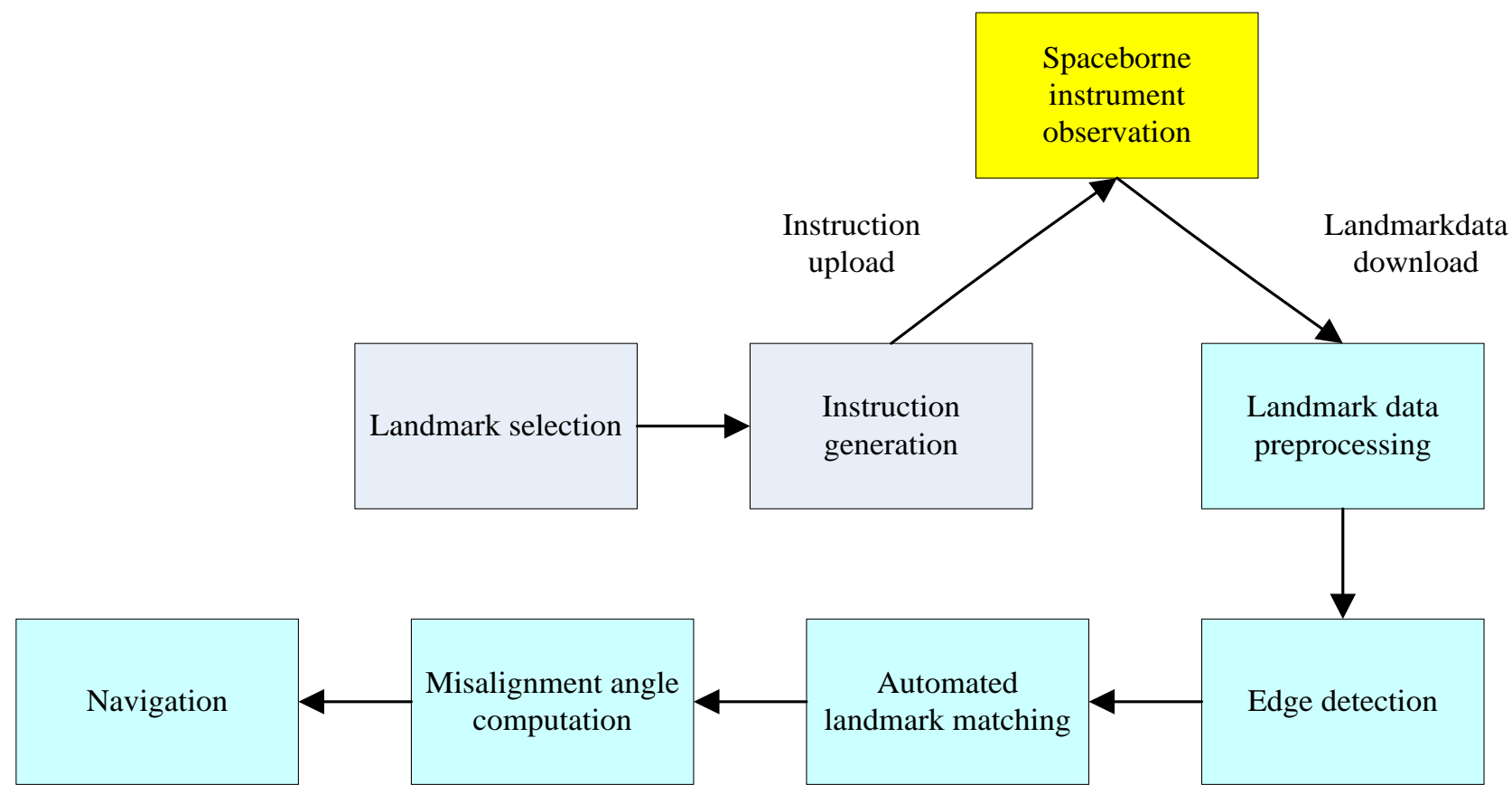

Figure 1. Processing diagram of landmark navigation. 
where $T_{\mathrm{ECI} 2 \mathrm{rb}}$ and $T_{\mathrm{ECR} 2 \mathrm{ECI}}$ are the rotating matrices from earth centered inertial coordinate system (ECI) to ORB and from ECR to ECI, respectively.

The view vector in instrument coordinate system (INST) is rotated to ORB.

$$
\boldsymbol{u}_{\mathrm{ORB}}=T_{\text {MISA }} T_{\text {SAT2ORB }} T_{\text {INST2SAT }} V_{\text {INST }}
$$

where $T_{\text {MISA }}$ is the misalignment matrix, $V_{\text {INST }}$ is the view vector in INST, $T_{\text {SAT2ORB }}$ and $T_{\text {INST2SAT }}$ are the rotating matrices from satellite coordinate system (SAT) to ORB and from INST to SAT, respectively. The former is attitude matrix, and the latter is mounting matrix.

Denote the misalignment angles of roll, yaw and pitch axis as $\xi_{r}, \xi_{y}, \xi_{p}$, respectively. The misalignment matrix is expressed as

$$
T_{\text {MISA }}=R_{z}\left(-\xi_{y}\right) R_{x}\left(-\xi_{r}\right) R_{y}\left(-\xi_{p}\right)
$$

where

$$
\begin{aligned}
& R_{x}(\theta)=\left[\begin{array}{ccc}
1 & 0 & 0 \\
0 & \cos \theta & \sin \theta \\
0 & -\sin \theta & \cos \theta
\end{array}\right], R_{y}(\theta)=\left[\begin{array}{ccc}
\cos \theta & 0 & -\sin \theta \\
0 & 1 & 0 \\
\sin \theta & 0 & \cos \theta
\end{array}\right], \\
& R_{z}(\theta)=\left[\begin{array}{ccc}
\cos \theta & \sin \theta & 0 \\
-\sin \theta & \cos \theta & 0 \\
0 & 0 & 1
\end{array}\right]
\end{aligned}
$$

and $\theta$ denotes $\xi_{r}, \xi_{y}, \xi_{p}$.

As long as the three angles are pretty small, the misalignment matrix, which is the most important information in three-axis stabilized geostationary satellite, can be written as

$$
T_{\mathrm{MISA}}=\left[\begin{array}{ccc}
1 & -\xi_{y} & \xi_{p} \\
\xi_{y} & 1 & -\xi_{r} \\
-\xi_{p} & \xi_{r} & 1
\end{array}\right]
$$

Using Equation (2) and the unit vector of Equation (1), the following equation can be obtained:

$$
\left[\begin{array}{ccc}
1 & -\xi_{y} & \xi_{p} \\
\xi_{y} & 1 & -\xi_{r} \\
-\xi_{p} & \xi_{r} & 1
\end{array}\right] T_{\text {SAT2ORB }} T_{\text {INST2SAT }} V_{\text {INST }}=\left[\begin{array}{l}
u_{x} \\
u_{y} \\
u_{z}
\end{array}\right]
$$

Equation (6) can be reorganized to get the final equation, based on which $\xi_{r}, \xi_{y}, \xi_{p}$ can be calculated.

$$
\begin{gathered}
{\left[\begin{array}{ccc}
0 & f_{3}(\alpha, \beta) & -f_{2}(\alpha, \beta) \\
-f_{3}(\alpha, \beta) & 0 & f_{1}(\alpha, \beta) \\
f_{2}(\alpha, \beta) & -f_{1}(\alpha, \beta) & 0
\end{array}\right]\left[\begin{array}{l}
\xi_{r} \\
\xi_{p} \\
\xi_{y}
\end{array}\right]=\left[\begin{array}{l}
u_{x}-f_{1}(\alpha, \beta) \\
u_{y}-f_{2}(\alpha, \beta) \\
u_{z}-f_{3}(\alpha, \beta)
\end{array}\right]} \\
{\left[\begin{array}{l}
f_{1}(\alpha, \beta) \\
f_{2}(\alpha, \beta) \\
f_{3}(\alpha, \beta)
\end{array}\right]=T_{\text {SAT 2ORB } T_{\text {INST } 2 S A T} V_{\text {INST }}}}
\end{gathered}
$$

where $\alpha$ and $\beta$ are the instrument's scan angle in east-west direction and 
step angle in north-south direction, respectively.

2) Star navigation

Although landmark navigation deals with the errors of the whole link of satellite-to-ground, the navigation accuracy is limited greatly by the accuracy of landmark matching and cloud cover. The primary steps of star navigation are proposed as follows, which is shown in Figure 2.

a) Predict stars according to given time and predicted satellite orbit.

b) Select proper stars using star observation strategy.

c) Generate observation instructions corresponding each selected landmark, and upload to the satellite.

d) The spaceborne instruments carry out observations of the stars, and data are transmitted to the ground.

e) Implement star center extraction and obtain the deviated position of each star.

f) Calculate misalignment angles using stars' deviations and ideal positions.

g) Finish accurate navigation calculation using misalignment angles, orbit data, attitude data, etc.

Denote a star's right ascension and declination as $\alpha_{0}$ and $\delta_{0}$, respectively, at a given time. The view vector of the star is

$$
\left[\begin{array}{c}
\cos \delta_{0} \cos \alpha_{0} \\
\cos \delta_{0} \sin \alpha_{0} \\
\sin \delta_{0}
\end{array}\right]
$$

The instrument view vector can be denoted by its pointing angles, corresponding to the target. Rotate the view vector from INST to ECI, and we can get

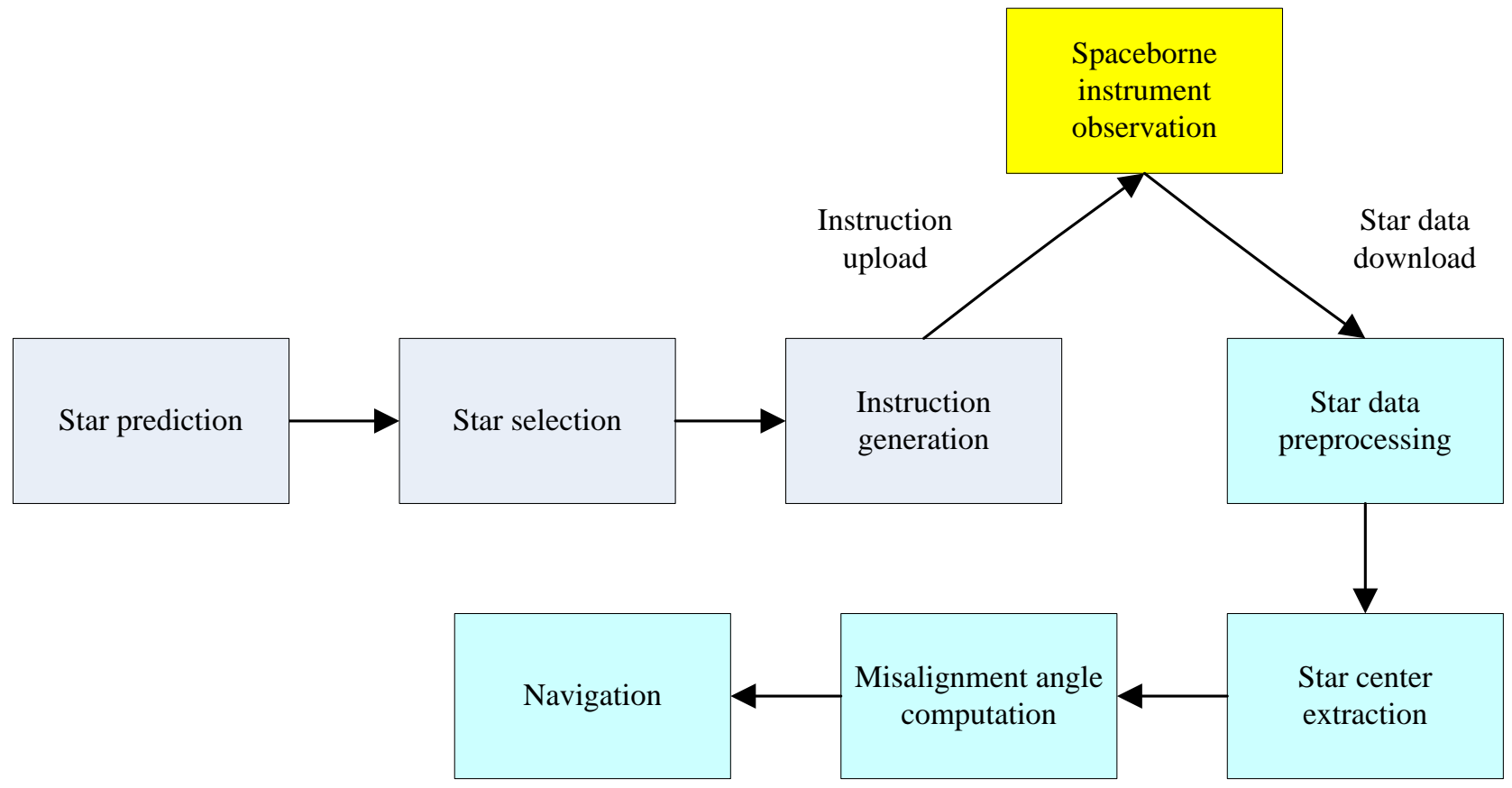

Figure 2. Processing diagram of star navigation. 


$$
T_{\text {ORB2ECI }} T_{\text {SAT2ORB }} T_{\text {INST2SAT }} V_{\text {INST }}=\left[\begin{array}{c}
\cos \delta_{0} \cos \alpha_{0} \\
\cos \delta_{0} \sin \alpha_{0} \\
\sin \delta_{0}
\end{array}\right]
$$

The misalignment angles can be treated as the attitude variation in ORB. So suppose three misalignment angles $\xi_{r}, \xi_{y}, \xi_{p}$ to represent the misalignment in roll, yaw and pitch direction, respectively. Equation (10) is changed to

$$
\begin{aligned}
& R_{z}\left(-\xi_{y}\right) R_{x}\left(-\xi_{r}\right) R_{y}\left(-\xi_{p}\right) T_{\mathrm{SAT} 2 \mathrm{ORB}} T_{\mathrm{INST} 2 \mathrm{ST}} V_{\mathrm{INST}} \\
& =T_{\mathrm{ORB} 2 \mathrm{ECI}}^{-1}\left[\begin{array}{c}
\cos \delta_{0} \cos \alpha_{0} \\
\cos \delta_{0} \sin \alpha_{0} \\
\sin \delta_{0}
\end{array}\right]=\left[\begin{array}{l}
u_{x} \\
u_{y} \\
u_{z}
\end{array}\right]
\end{aligned}
$$

and

$$
\left[\begin{array}{ccc}
1 & -\xi_{y} & \xi_{p} \\
\xi_{y} & 1 & -\xi_{r} \\
-\xi_{p} & \xi_{r} & 1
\end{array}\right] T_{\mathrm{SAT2ORB}} T_{\mathrm{INST2SAT}} V_{\mathrm{INST}}=\left[\begin{array}{l}
u_{x} \\
u_{y} \\
u_{z}
\end{array}\right]
$$

After transformation, Equation (13) can be obtained, which is the basis of misalignment angle calculation.

$$
\begin{gathered}
{\left[\begin{array}{ccc}
0 & f_{3}(\alpha, \beta) & -f_{2}(\alpha, \beta) \\
-f_{3}(\alpha, \beta) & 0 & f_{1}(\alpha, \beta) \\
f_{2}(\alpha, \beta) & -f_{1}(\alpha, \beta) & 0
\end{array}\right]\left[\begin{array}{l}
\xi_{r} \\
\xi_{p} \\
\xi_{y}
\end{array}\right]=\left[\begin{array}{l}
u_{x}-f_{1}(\alpha, \beta) \\
u_{y}-f_{2}(\alpha, \beta) \\
u_{z}-f_{3}(\alpha, \beta)
\end{array}\right]} \\
{\left[\begin{array}{l}
f_{1}(\alpha, \beta) \\
f_{2}(\alpha, \beta) \\
f_{3}(\alpha, \beta)
\end{array}\right]=T_{\mathrm{SAT2ORB}} T_{\text {INST2SAT }} V_{\text {INST }}}
\end{gathered}
$$

\subsection{Experimental Data Construction and Analyzing Method}

Before the satellite is launched, there are no observation data which can be used to go through navigation procedures and estimate navigation accuracy. Without these analyses, image navigation of three-axis stabilized geostationary satellite exists risks. Under this condition, we try to construct experimental data as follows.

For landmark navigation and corresponding misalignment calculation:

1) Design different attitude disturbance.

2) Calculate land-sea-masks by means of navigation processing, including nominal land-sea-mask and land-sea-masks corresponding to each attitude disturbance.

3) Implement automatic landmark matching between each land-sea-mask with attitude disturbance and nominal land-sea-mask, and obtain the matching results.

4) Calculate misalignment angles using misalignment model.

5) Compare the results with the designed disturbances, and evaluate misalignment modeling and calculation accuracy. 
6) To go a step further, redo image navigation using the misalignment angles, and output corresponding land-sea-masks.

7) Implement automatic landmark matching between land-sea-masks obtained from (f) and land-sea-masks obtained from (b), and output the matching results.

8) Analyze the results of ( $g$ ) and evaluate navigation accuracy.

Note that the landmarks used in (g) are different from those used in (c). The procedures are shown in Figure 3.

For star navigation and corresponding misalignment calculation:

1) Design different attitude disturbance.

2) Specify observation angles of different stars.

3) By means of coordinate system transformation, calculate each star's position in observation images; this procedure should use the misalignment matrix.

4) By means of coordinate system transformation, calculate each star's right ascension and declination, using each star's observation angles.

5) Calculate misalignment angles using each star's right ascension, declination, position in observation images, orbit data, attitude data, etc.

6) Compare the misalignment calculation results with the designed attitude disturbance, and evaluate the accuracy of misalignment calculation.

The procedures of experimental data construction and misalignment calculation accuracy analysis are shown in Figure 4.

\section{Results}

\subsection{Landmark Navigation}

The simulated experimental data are shown in Figure 5. Figure 5(a) shows nominal land-sea-mask overlapped with small attitude disturbance, while Figure 5(b) with large disturbance. The attitude disturbance represents the thermal

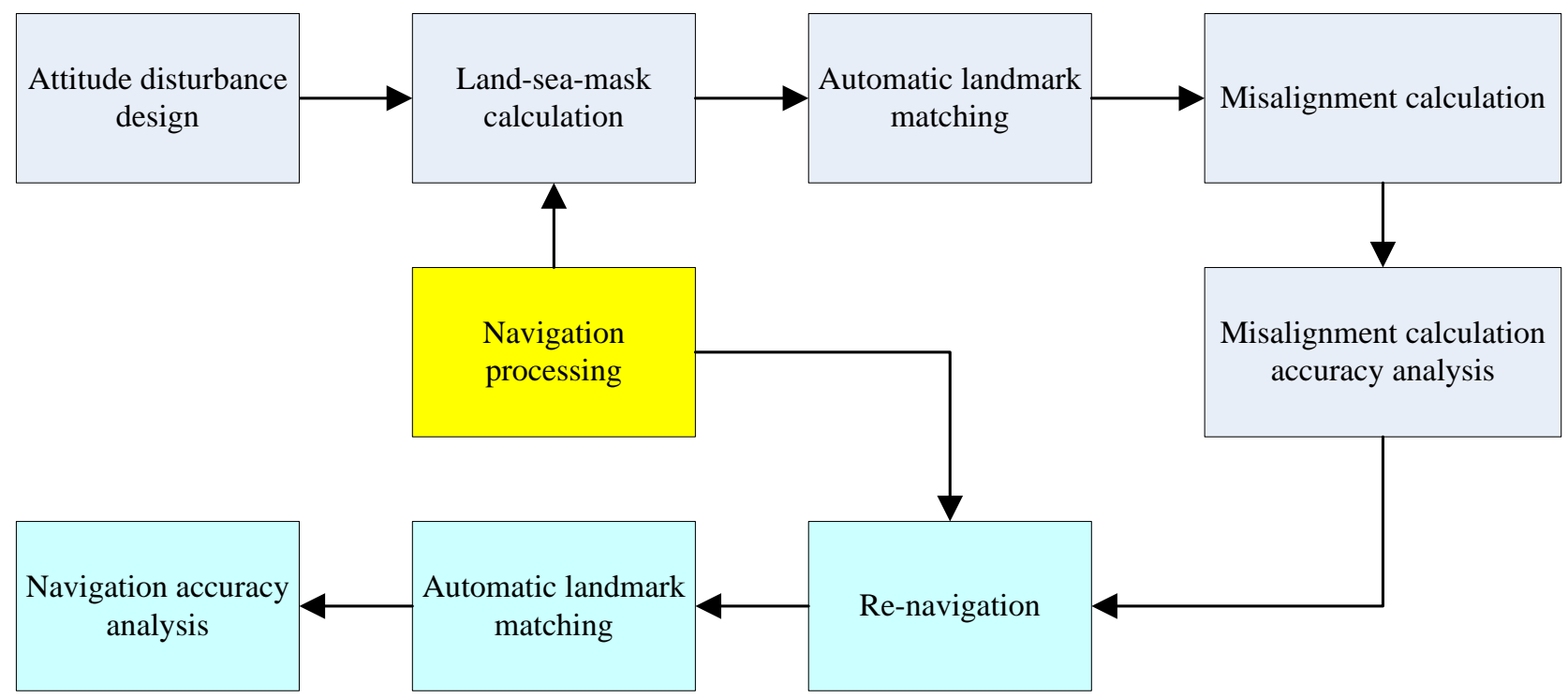

Figure 3. Procedures of experimental data construction and accuracy analysis of landmark navigation. 


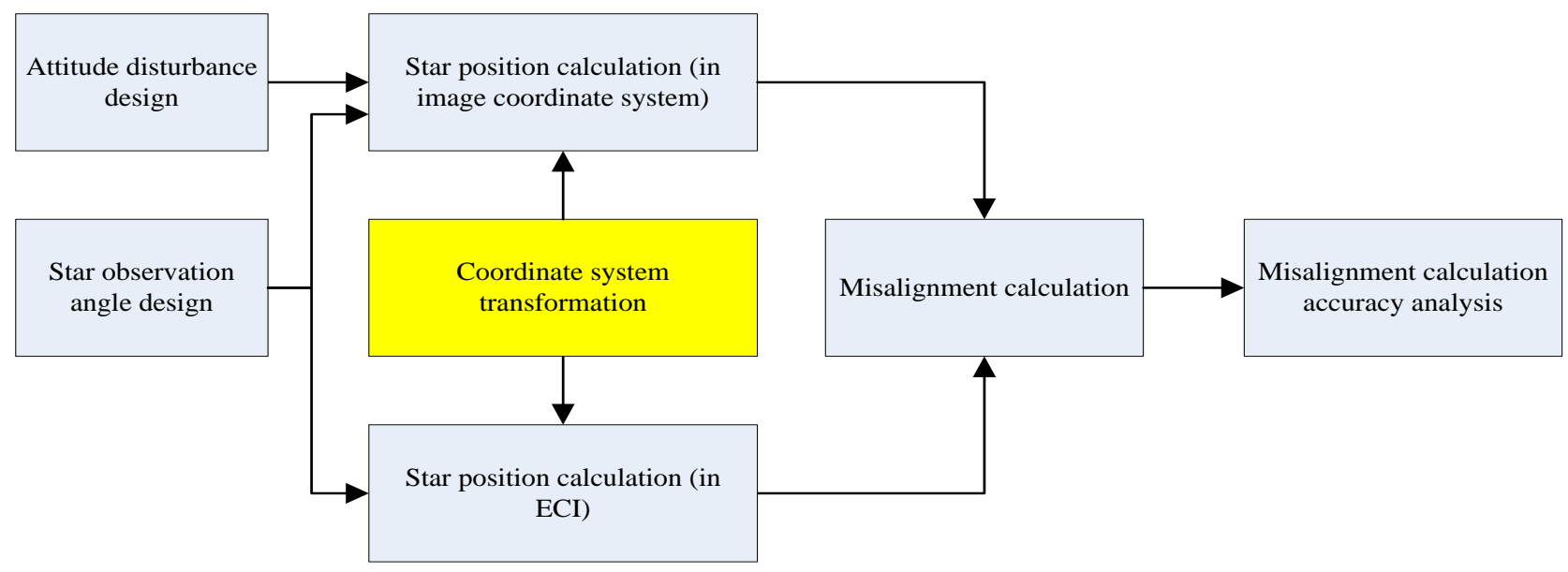

Figure 4. Procedures of experimental data construction and accuracy analysis of star navigation.

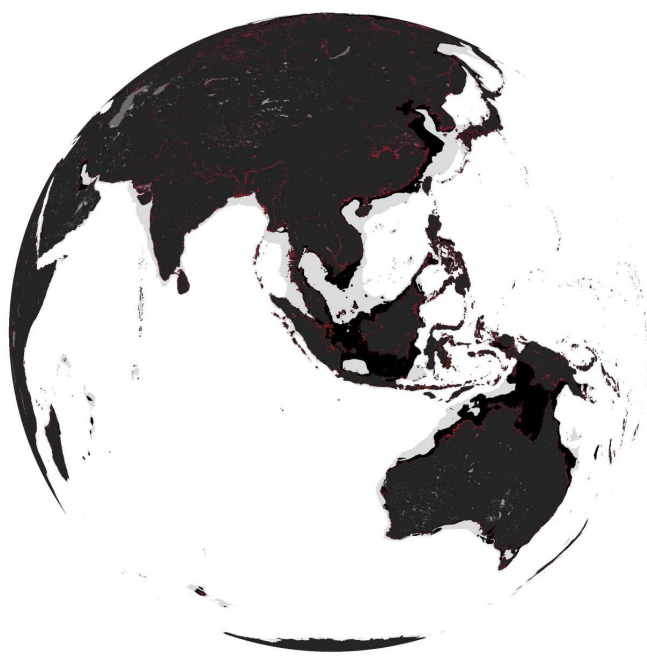

(a)

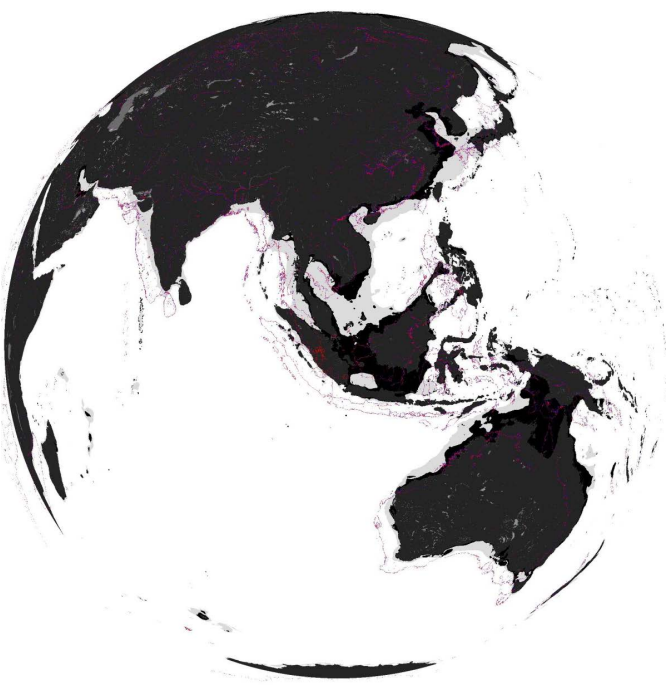

(b)

Figure 5. Nominal land-sea-mask overlapped with attitude disturbance. (a) Small attitude disturbance; (b) Large attitude disturbance. 
elastic distortion here. The thermal elastic distortion can reach into hundreds of micro radians on the GOES instruments [4]. The designed attitude disturbances range from 30 to 810 micro radians. Data are processed using the method presented in part 2.2, and the detailed results are given in Table 1. The inputs include disturbances added to roll, pitch, and yaw axes, and important procedures are navigation calculation, automatic landmark matching, and misalignment angle calculation. The outputs include landmark deviations and misalignment calculation errors, which are the differences of the calculation results and the simulated attitude disturbance. The misalignment angle errors of roll axis and pitch axis both range from 0 to 2 micro radians, which are rather small, although there are fluctuations. The misalignment angle errors of yaw axis range from 3 to 104 micro radians. Although the calculation error of yaw axis is larger than the other two axes, the overall results are satisfying for geostationary satellites. The main reason of yaw axis error is from the error of automatic landmark matching, which is difficult to indicate the rotation while attitude disturbance is not very large.

To go a step further, image navigation is redone using the calculated misalignment angles, and the new landmark matching results are the most useful information in navigation accuracy analysis. This time the automatic landmark matching should be carried out between land-sea-masks obtained from re-navigation and land-sea-masks obtained from original navigation. Several cases of land-sea-masks with attitude disturbances overlapped with re-navigated land-sea-masks are given in Figure 6, in which Australian northern coast and China's Bohai Bay are

Table 1. Misalignment calculation results of landmarks (unit: micro radians).

\begin{tabular}{|c|c|c|c|c|c|c|}
\hline No. & $\begin{array}{c}\text { Roll } \\
\text { disturbance }\end{array}$ & $\begin{array}{c}\text { Pitch } \\
\text { disturbance }\end{array}$ & $\begin{array}{c}\text { Yaw } \\
\text { disturbance }\end{array}$ & $\begin{array}{l}\text { Landmark } \\
\text { matching } \\
(\Delta \mathrm{i} / \Delta \mathrm{j})\end{array}$ & $\begin{array}{l}\text { Misalignment } \\
\text { angles } \\
\text { (roll/pitch/yaw) }\end{array}$ & $\begin{array}{l}\text { Misalignment } \\
\text { calculation } \\
\text { error }\end{array}$ \\
\hline 1 & 30 & 30 & 30 & $0 / 0$ & $30 / 30 / 2$ & $0 / 0 /-28$ \\
\hline 2 & 90 & 90 & 90 & $0 / 0.3$ & $90 / 90 / 4$ & $0 / 0 /-86$ \\
\hline 3 & 150 & 150 & 150 & $-0.6 / 0.6$ & $150 / 149 / 46$ & $0 /-1 /-104$ \\
\hline 4 & 210 & 210 & 210 & $-2.7 / 5.4$ & $211 / 208 / 219$ & $1 /-2 / 9$ \\
\hline 5 & 270 & 270 & 270 & $-3.3 / 9$ & $272 / 269 / 296$ & $2 /-1 / 26$ \\
\hline 6 & 330 & 330 & 330 & $-5.7 / 11.7$ & $330 / 330 / 335$ & $0 / 0 / 5$ \\
\hline 7 & 390 & 390 & 390 & $-7.5 / 13.8$ & $389 / 391 / 378$ & $-1 / 1 /-12$ \\
\hline 8 & 450 & 450 & 450 & $-9.3 / 16.8$ & $448 / 449 / 441$ & $-2 /-1 /-9$ \\
\hline 9 & 510 & 510 & 510 & $-10.8 / 19.5$ & $508 / 511 / 518$ & $-2 / 1 / 8$ \\
\hline 10 & 570 & 570 & 570 & $-10.5 / 21.3$ & $569 / 571 / 577$ & $-1 / 1 / 7$ \\
\hline 11 & 630 & 630 & 630 & $-9.9 / 23.1$ & $631 / 631 / 633$ & $1 / 1 / 3$ \\
\hline 12 & 690 & 690 & 690 & $-10.8 / 23.7$ & $691 / 690 / 679$ & $1 / 0 /-11$ \\
\hline 13 & 750 & 750 & 750 & $-12.3 / 26.7$ & $750 / 751 / 743$ & $0 / 1 /-7$ \\
\hline 14 & 810 & 810 & 810 & $-13.5 / 30$ & $810 / 811 / 813$ & $0 / 1 / 3$ \\
\hline
\end{tabular}



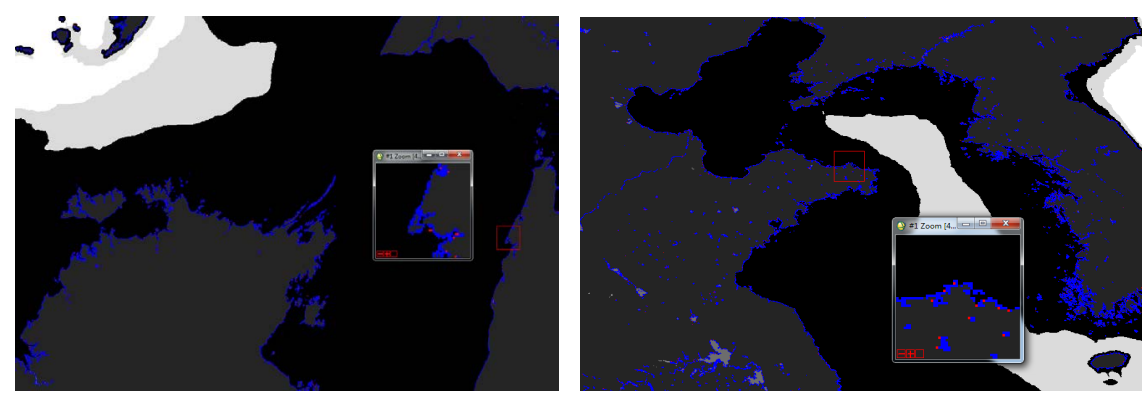

(a)
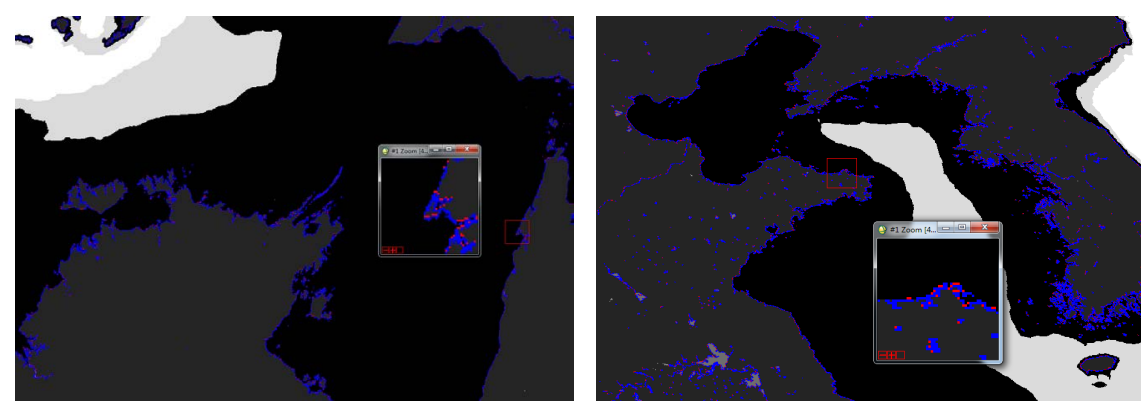

(b)
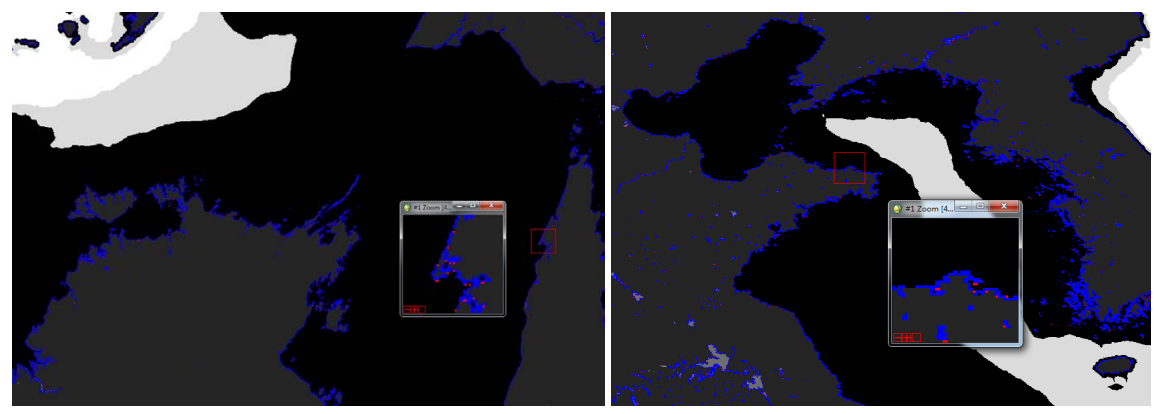

(c)
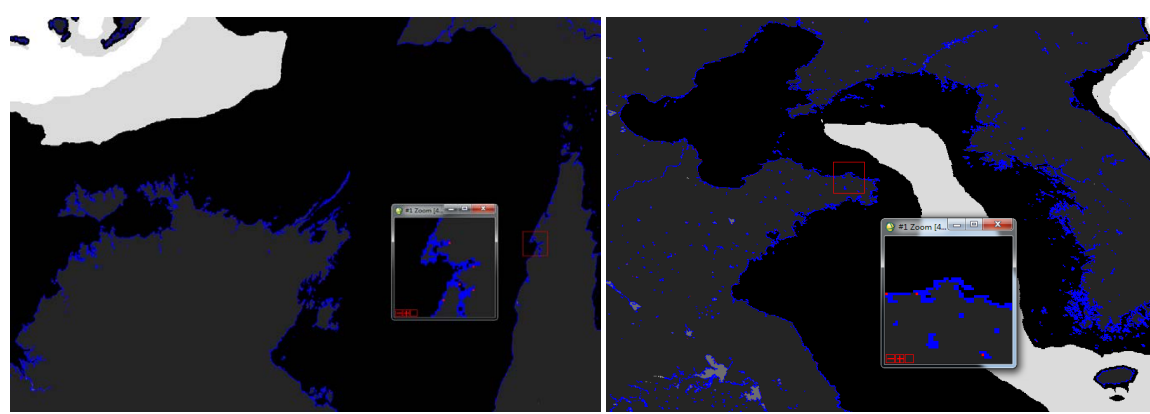

(d)

Figure 6. Land-sea-mask with attitude disturbance overlapped with re-navigated land-sea-mask. (a) 30 micro radians disturbance; (b) 150 micro radians disturbance; (c) 510 micro radians disturbance; (d) 810 micro radians disturbance.

shown. The former is expressed in red, while the latter is expressed in blue. Almost only blue lines can be seen in Figure 6, which demonstrates the re-navigation is quite accurate. Landmark matching results are given in Table 2, whose re-navigation results are rather good, with almost all the landmarks' deviations are zero. Four examples are shown because of length limitation, including small, 
Table 2. Re-navigation results.

\begin{tabular}{cccc}
\hline No. & $\begin{array}{c}\text { Attitude disturbance } \\
\text { (micro radians) }\end{array}$ & Landmark deviation & Coastline figure \\
\hline 1 & 30 & All: 0 & Figure 5(a) \\
3 & 150 & 4 landmarks' deviations are 1, others are all 0 & Figure 5(b) \\
9 & 510 & All: 0 & Figure 5(c) \\
14 & 810 & All: 0 & Figure 5(d) \\
\hline
\end{tabular}

medium and large attitude disturbance, and other results are similar. This means the calculation of misalignment angles as well as the whole navigation procedures are practicable and effective.

\subsection{Star Navigation}

As misalignment angle calculation needs at least three stars' information, analysis is carried out in the case of three, four and five stars. In these different cases, the maximum misalignment angle calculation errors are less than 1, 3 and $2 \mathrm{mi}-$ cro radians, respectively. The maximum misalignment angle calculation errors of roll, pitch, and yaw axis are less than 1, 1, and 3 micro radians, respectively. Although the error of yaw axis is larger than the other two axes, it is still small enough. And the results are pretty stable. Table 3 shows the detailed simulation results. These results demonstrate that the misalignment modeling using stars and calculation are more accurate than using landmarks, which confirms the statement as mentioned above, as the accuracy of star position is higher than 0.1 milliarcseconds and the accuracy of star centroid extraction is higher than that of landmarks. In the face of the challenges in image navigation brought by three-axis stabilized attitude control mode in geostationary orbit, star navigation is indispensable.

\section{Conclusion}

Three-axis stabilized attitude control mode is the development trend of geostationary satellites for meteorology, resources, communications, and military applications. In this background, the great difficulty of image navigation must be faced and overcome. Landmark navigation and star navigation methods are both considered and developed. The important aspects affecting navigation accuracy include misalignment modeling \& calculation accuracy, landmark matching accuracy and star center extraction accuracy. In this paper, misalignment modeling of landmark navigation and star navigation is introduced, and misalignment angles are calculated using experimental data we constructed. The misalignment calculation accuracy is rather satisfying, especially using stars. The misalignment modeling as well as calculation method has been used in the new generation of geostationary meteorological satellite in China, FY-4 series, the first satellite of which was launched at December, 2016, and obtained satisfactory navigation results during the in-orbit test. 
Table 3. Misalignment calculation results of stars.

\begin{tabular}{|c|c|c|c|c|c|c|c|c|c|c|c|c|c|}
\hline \multirow[b]{2}{*}{ No. } & \multicolumn{2}{|c|}{ Star 1} & \multicolumn{2}{|c|}{ Star 2} & \multicolumn{2}{|c|}{ Star 3} & \multicolumn{2}{|c|}{ Star 4} & \multicolumn{2}{|c|}{ Star 5} & \multirow{2}{*}{ 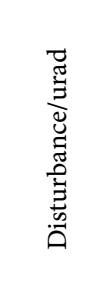 } & \multirow[b]{2}{*}{ 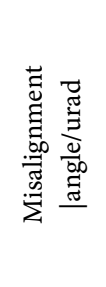 } & \multirow{2}{*}{ 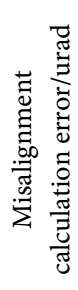 } \\
\hline & 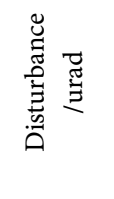 & 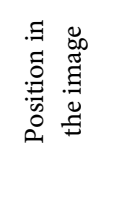 & 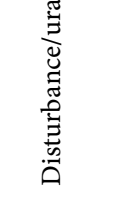 & 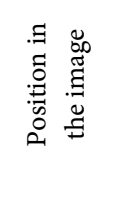 & 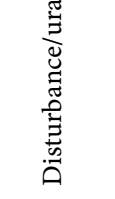 & 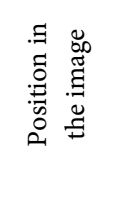 & 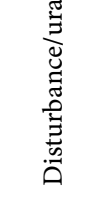 & 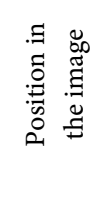 & 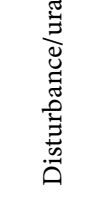 & 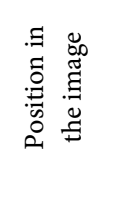 & & & \\
\hline \multirow{3}{*}{$3-1$} & -0.04 & $\mathrm{i}: 255.57$ & -0.19 & $\mathrm{i}: 254.91$ & -0.33 & i: 255.65 & & & & & -0.19 & -0.22 & -0.04 \\
\hline & -0.04 & $\mathrm{j}: 257.90$ & -0.19 & $\mathrm{j}: 267.46$ & -0.33 & $\mathrm{j}: 277.08$ & - & - & - & - & -0.19 & -0.20 & -0.01 \\
\hline & -0.02 & & -0.11 & & -0.20 & & & & & & -0.11 & -0.77 & -0.66 \\
\hline \multirow{3}{*}{$3-2$} & -58.81 & $\mathrm{i}: 253.72$ & -58.95 & $\mathrm{i}: 252.79$ & -59.10 & $\mathrm{i}: 253.72$ & & & & & -58.95 & -59.00 & -0.04 \\
\hline & -58.81 & $j: 259.67$ & -58.95 & $\mathrm{j}: 269.12$ & -59.10 & $\mathrm{j}: 279.22$ & - & - & - & - & -58.95 & -58.97 & -0.02 \\
\hline & -35.29 & & -35.37 & & -35.46 & & & & & & -35.37 & -36.03 & -0.66 \\
\hline \multirow{3}{*}{$3-3$} & -353.58 & i:244.46 & -353.68 & $\mathrm{i}: 242.13$ & -353.79 & i: 244.08 & & & & & -353.68 & -353.76 & -0.08 \\
\hline & -353.58 & $\mathrm{j}: 268.59$ & -353.68 & $\mathrm{j}: 277.44$ & -353.79 & j:289.98 & - & - & - & - & -353.68 & -353.72 & -0.04 \\
\hline & -212.15 & & -212.21 & & -212.27 & & & & & & -212.21 & -212.79 & -0.58 \\
\hline \multirow{3}{*}{$3-4$} & -499.94 & $\mathrm{i}: 239.87$ & -499.94 & i: 236.85 & -499.94 & i:239.29 & & & & & -499.94 & -500.04 & -0.10 \\
\hline & -499.94 & $\mathrm{j}: 273.02$ & -499.94 & $\mathrm{j}: 281.56$ & -499.94 & $\mathrm{j}: 295.32$ & - & - & - & - & -499.94 & -499.96 & -0.02 \\
\hline & -299.96 & & -299.96 & & -299.97 & & & & & & -299.96 & -300.20 & -0.24 \\
\hline \multirow{3}{*}{$4-1$} & -0.04 & $\mathrm{i}: 255.57$ & -0.19 & $\mathrm{i}: 254.91$ & -0.33 & i: 255.65 & -0.48 & i: 254.45 & & & -0.26 & -0.26 & -0.01 \\
\hline & -0.04 & j:257.90 & -0.19 & $\mathrm{j}: 267.46$ & -0.33 & $\mathrm{j}: 277.08$ & -0.48 & $\mathrm{j}: 286.66$ & - & - & -0.26 & -0.52 & -0.26 \\
\hline & -0.02 & & -0.11 & & -0.20 & & -0.29 & & & & -0.15 & -2.60 & -2.45 \\
\hline \multirow{3}{*}{$4-2$} & -58.81 & $\mathrm{i}: 253.72$ & -58.95 & $\mathrm{i}: 252.79$ & -59.10 & i: 253.72 & -59.24 & i: 252.35 & & & -59.03 & -59.04 & -0.01 \\
\hline & -58.81 & $\mathrm{j}: 259.67$ & -58.95 & $\mathrm{j}: 269.12$ & -59.10 & $\mathrm{j}: 279.22$ & -59.24 & $\mathrm{j}: 288.69$ & - & - & -59.03 & -59.29 & -0.27 \\
\hline & -35.29 & & -35.37 & & -35.46 & & -35.55 & & & & -35.42 & -37.86 & -2.45 \\
\hline \multirow{3}{*}{$4-3$} & -353.58 & i:244.46 & -353.68 & $\mathrm{i}: 242.13$ & -353.79 & i: 244.08 & -353.89 & i: 241.84 & & & -353.74 & -353.79 & -0.05 \\
\hline & -353.58 & $\mathrm{j}: 268.59$ & -353.68 & $\mathrm{j}: 277.44$ & -353.79 & $\mathrm{j}: 289.98$ & -353.89 & & - & - & -353.74 & -354.03 & -0.30 \\
\hline & -212.15 & & -212.21 & & -212.27 & & -212.33 & $\mathrm{j}: 298.90$ & & & -212.24 & -214.54 & -2.30 \\
\hline \multirow{3}{*}{$4-4$} & -499.94 & i:239.87 & -499.94 & $\mathrm{i}: 236.85$ & -499.94 & i:239.29 & -499.95 & i: 236.63 & & & -499.94 & -500.02 & -0.08 \\
\hline & -499.94 & $\mathrm{j}: 273.02$ & -499.94 & $\mathrm{j}: 281.56$ & -499.94 & $\mathrm{j}: 295.32$ & -499.95 & $\mathrm{j}: 303.96$ & - & - & -499.94 & -500.24 & -0.30 \\
\hline & -299.96 & & -299.96 & & -299.97 & & -299.97 & & & & -299.97 & -301.74 & -1.78 \\
\hline \multirow{3}{*}{$5-1$} & -0.04 & $\mathrm{i}: 255.57$ & -0.19 & $\mathrm{i}: 254.91$ & -0.33 & i: 255.65 & -0.48 & i: 254.45 & -0.62 & i: 256.94 & -0.33 & -0.37 & -0.03 \\
\hline & -0.04 & $\mathrm{j}: 257.90$ & -0.19 & j:267.46 & -0.33 & $\mathrm{j}: 277.08$ & -0.48 & $\mathrm{j}: 286.66$ & -0.62 & $\mathrm{j}: 296.19$ & -0.33 & -0.33 & -0.00 \\
\hline & -0.02 & & -0.11 & & -0.20 & & -0.29 & & -0.37 & & -0.20 & -1.31 & -1.11 \\
\hline \multirow{3}{*}{$5-2$} & -58.81 & $\mathrm{i}: 253.72$ & -58.95 & $\mathrm{i}: 252.79$ & -59.10 & $\mathrm{i}: 253.72$ & -59.24 & i: 252.35 & -59.39 & $\mathrm{i}: 255.14$ & -59.10 & -59.14 & -0.04 \\
\hline & -58.81 & j:259.67 & -58.95 & $\mathrm{j}: 269.12$ & -59.10 & $\mathrm{j}: 279.22$ & -59.24 & $\mathrm{j}: 288.69$ & -59.39 & $\mathrm{j}: 298.41$ & -59.10 & -59.10 & -0.01 \\
\hline & -35.29 & & -35.37 & & -35.46 & & -35.55 & & -35.63 & & -35.46 & -36.57 & -1.11 \\
\hline \multirow{3}{*}{$5-3$} & -353.58 & i: 244.46 & -353.68 & $\mathrm{i}: 242.13$ & -353.79 & i: 244.08 & -353.89 & i: 241.84 & -353.99 & i: 246.16 & -353.79 & -353.87 & -0.08 \\
\hline & -353.58 & $\mathrm{j}: 268.59$ & -353.68 & $\mathrm{j}: 277.44$ & -353.79 & j:289.98 & -353.89 & $\mathrm{j}: 298.90$ & -353.99 & $\mathrm{j}: 309.54$ & -353.79 & -353.83 & -0.04 \\
\hline & -212.15 & & -212.21 & & -212.27 & & -212.33 & & -212.40 & & -212.27 & -213.23 & -0.96 \\
\hline \multirow{3}{*}{$5-4$} & -499.94 & i:239.87 & -499.94 & $\mathrm{i}: 236.85$ & -499.94 & i:239.29 & -499.95 & i: 236.63 & -499.95 & i: 241.71 & -499.94 & -500.04 & -0.10 \\
\hline & -499.94 & $\mathrm{j}: 273.02$ & -499.94 & $\mathrm{j}: 281.56$ & -499.94 & $\mathrm{j}: 295.32$ & -499.95 & $\mathrm{j}: 303.96$ & -499.95 & $\mathrm{j}: 315.05$ & -499.94 & -500.01 & -0.06 \\
\hline & -299.96 & & -299.96 & & -299.97 & & -299.97 & & -299.97 & & -299.97 & -300.42 & -0.46 \\
\hline
\end{tabular}




\section{Acknowledgements}

This work was supported by the National Natural Science Foundation of China

(Grant No. 61172113 and 91338109).

\section{References}

[1] NOAA and NASA (2005) GOES N Data Book (Rev B). NASA, Washington DC.

[2] Lu, F., Zhang, X. and Xu, J. (2008) Image Navigation for the FY2 Geostationary Meteorological Satellite. Journal of Atmospheric and Oceanic Technology, 25, 1149-1165. https://doi.org/10.1175/2007JTECHA964.1

[3] Harris, J. and Just, D. (2010) INR Performance Simulations for MTG. SpaceOps 2010 Conference of the American Institute of Aeronautics and Astronautics, Alabama, 25-30 April 2010. https://doi.org/10.2514/6.2010-2322

[4] Carr, J. (2009) Twenty-Five Years of INR. Journal of the Astronautical Sciences, 57, 505-515. https://doi.org/10.1007/BF03321514

[5] Li, Q. and Dong, Y. (2008) Achievement and Forecast of Meteorological Satellite Technology in China. Aerospace Shanghai, 25, 1-10.

[6] U.S. DOC, NOAA, NESDIS and NASA (2014) Product Definition and Users' Guide (PUG) Volume 5A: Level 2+ Products. NOAA, Washington DC.

[7] Yang, L. and Yang, Z. (2009) Automatic Image Navigation Method for Remote Sensing Satellite. Computer Engineering and Applications, 45, 204-207.

[8] Yang, L., Feng, X., Lv, K. and Shang, J. (2014) Automated Landmark Matching of FY-2 Visible Imagery with Its Applications to the On-Orbit Image Navigation Performance Analysis and Improvements. Chinese Journal of Electronics, 23, 649-654.

[9] Yang, L. and Shang, J. (2011) Modeling and Computation on the Attitude Misalignment Parameters for Geostationary Meteorological Satellite. Chinese Journal of Electronics, 20, 370-374. 